\title{
Causal Slingshots
}

\author{
Michael Baumgartner
}

Received: 1 June 2008/Accepted: 7 August 2009/Published online: 14 November 2009

(C) Springer Science+Business Media B.V. 2009

\begin{abstract}
Causal slingshots are formal arguments advanced by proponents of an event ontology of token-level causation which, in the end, are intended to show two things: (i) The logical form of statements expressing causal dependencies on token level features a binary predicate "... causes ..." and (ii) that predicate takes events as arguments. Even though formalisms are only revealing with respect to the logical form of natural language statements, if the latter are shown to be adequately captured within a corresponding formalism, proponents of slingshots usually take the adequacy of their formalizations for granted without justifying it. The first part of this paper argues that the most discussed version of a causal slingshot, viz. the one e.g. presented by Davidson (Essays on actions and events. Oxford, Clarendon Press, 1980), can indeed be refuted for relying on an inadequate formal apparatus. In contrast, the formal means of Gödel's (The philosophy of Betrand Russell. New York, Tudor, 1944) often neglected slingshot are shown to stand on solid ground in the second part of the paper. Nonetheless, I contend that Gödel's slingshot does only half the work friends of event causation would like it to do. It provides good reasons for (i) but not for (ii).
\end{abstract}

\section{Introduction}

The question as to what entities constitute the relata of singular causation, i.e. causation on token level, has been controversially debated in the literature of the past 50 years. Among the many different entities or categories that have been brought into play, two have received the widest reception: events and facts. Many

\footnotetext{
M. Baumgartner $(\bowtie)$

Department of Philosophy, University of Bern, Länggassstrasse 49a, 3012 Bern, Switzerland e-mail: baumgartner@philo.unibe.ch
} 
arguments in favor or against event and fact theories of causation essentially turn on the question as to which is the proper logical analysis of statements expressing causal dependencies on token level. Are such dependencies expressed by means of the relation "... causes/d ..." that takes events as arguments, or, rather, by means of the sentential connective "The fact that ... causes/d the fact that ..." that concatenates fact reporting statements? ${ }^{1}$

Both answers to that question have their advantages and disadvantages. ${ }^{2}$ While it is difficult to account for causal dependencies among absences or omissionswhich are not normally typecast as events-within an event framework, ${ }^{3}$ the main problem for a fact ontology of singular causation stems from a collapsing argument which Barwise and Perry (1996, p. 375) have famously labeled the slingshot argument as "[t]he argument is so small, seldom encompassing more than half a page, and employs such a minimum of ammunition". The slingshot argument, or rather-since there is a whole series of such arguments - the slingshot arguments, originally are not tailored against the analysis of token-level causes and effects as facts, but against philosophical recourse to facts in general. Concisely put, these arguments yield the paradoxical result that non-extensional (non-truth-functional) sentential connectives linking statements that stand for or express extralinguistic entities - as facts or states of affairs - turn out to be extensional after all, provided that two seemingly unproblematic inference principles are taken to hold for these connectives. Roughly, the first of these principles allows for a truth-conserving substitution of logically equivalent expressions and the second licenses the substitution of co-referring singular terms within contexts governed by a pertaining connective.

Slingshots have most often been raised against the connective "The fact that ... is identical to the fact that ...". When applied to this connective, slingshot arguments are taken to demonstrate that any fact is identical to any fact, thus, that there is at most one fact. However, if the question as to the identity of facts is raised in isolation, the fact theorist can easily counter a corresponding slingshot by stipulating that facts are fine-grained entities whose identity depends on the predicates and singular terms used to state them. Accordingly, one or both of the inference rules employed in such slingshots may easily be dismissed for the fact identity connective-as e.g. emphatically done by Oppy (1997). Yet, as soon as facts are advanced as token causes and effects, this way around slingshot arguments becomes much more problematic, because the validity of these two inference principles for factual causal statements is very much backed by causal intuitions. The following inferences are informally valid:

\footnotetext{
${ }^{1}$ While Davidson (1980) is a typical proponent of the first analysis, Mellor (1991), for instance, prefers the second option.

2 In consequence, authors such as Bennett (1988) or Dowe (2000) argue that both events and facts can, depending on the context, function as causes and effects.

${ }^{3}$ Cf. e.g. Mellor (1991) and Mellor (1995, pp. 131-135).
} 
(i) The fact that Brutus stabbed Caesar caused the fact that Caesar died. Brutus stabbed Caesar if and only if Brutus did not not stab Caesar.

The fact that Brutus did not not stab Caesar caused the fact that Caesar died.

(ii) The fact that Brutus stabbed Caesar caused the fact that Caesar died. Brutus is identical with the son of Servilia Caepionis.

The fact that the son of Servilia Caepionis stabbed Caesar caused the fact that Caesar died.

Relative to all reinterpretations of the non-logical vocabulary embedded in the factual contexts of these arguments it holds that if the premises are judged to be true, so are the conclusions. Intuitively, causal dependencies subsist in nature and are independent of what logically equivalent expressions or co-referring singular terms are chosen to express them. Moreover, the causes of the fact that Caesar died mentioned in the first premises of (i) and (ii) are identical to the causes of that same effect mentioned in the conclusions of (i) and (ii). To the informal validity of (i) and (ii) and the identity of corresponding causes I shall in the following refer as the robustness of singular causation. Yet, as indicated above, given the validity of both inference principles instantiated in (i) and (ii), slingshot arguments yield that complex statements governed by "The fact that ... causes/d the fact that ..." are truth-functional, which, in effect, they certainly are not. Furthermore, if slingshots are directed against factual causal statements, it turns out that any effect must be taken to be caused by any fact. Hence, by advancing causal slingshots proponents of event causation intend to confront their rivals of the fact causation camp with the following dilemma: either (A) their accounts imply that any fact trivially causes any other fact or (B) they are forced to stipulate that at least one of the inference principles instantiated in (i) and (ii) is invalid for causal statements, which means that fact theories do not adequately capture the robustness of singular causation.

All the different variants of causal slingshots have one thing in common: they presuppose a very specific kind of syntax that treats particular formal expressions as primitive symbols, viz. expressions governed by class abstraction or iota-operators. They are thus formal arguments to the effect that statements expressing causal dependencies are of one logical form rather than another. Before formalisms can be put to work when it comes to answering the question as to the logical form of causal statements, the latter must be transformed into corresponding formalisms. Such transformations call for stringent justification (cf. Massey 1981, pp. 17-18). Unfortunately though, advocates of causal slingshots normally take the adequacy of their formalizations for granted without justifying it. This neglect, as the first part of the paper at hand intends to show, is especially unsatisfactory because the debate over the consequences of slingshots for the ontology of singular causation has commonly focused on one specific slingshot variant-most famously put forward by Davidson $(1980)^{4}$ - which the friend of fact causation can indeed reject for its

\footnotetext{
${ }^{4}$ That is not to say that discussions about non-causal variants of slingshot arguments have also centered around Davidson's argument. Especially since the early nineties the work of Stephen Neale has shifted
} 
reliance on inadequate formalizations without being compelled to accept either horn of the slingshot dilemma.

Matters are different if we turn to another variant of a slingshot. In (1995) and (2001) Neale has recalled attention to an often neglected slingshot argument that is due to Gödel (1944). The second part of this paper is going to demonstrate that the adequacy of the formal means employed by Gödel is much less easily challenged. While it is possible to both refute Davidson's slingshot and avoid the slingshot dilemma, the fact theorist challenging the formal apparatus of Gödel's argument runs into horn (B) of that dilemma. The Gödelian slingshot hence provides strong reasons in favor of logically analyzing statements expressing singular causation in terms of atomic statements featuring the binary predicate "... causes/d ...". The paper concludes by arguing that this constraint imposed on the logical analysis of causal statements does not, as the event theorist would like to have it, render a fact ontology of causation impossible. Nonetheless, it calls for a non-standard analysis of factual causal statements.

\section{Events Versus Facts}

The notions of an event and a fact are often blended in the literature. ${ }^{5}$ Accordingly, before we can look at causal slingshots, a minimal understanding of the ontological difference between events and facts is required. To this end, I shall here presume the following minimal contrast between events and facts, for which Ramsey (1927/ 1994) has most notably argued: events are particulars-simple or complex-to which reference is made by means of singular terms, i.e. proper names or referentially understood definite descriptions, whereas facts are expressed, represented or stated by closed sentences (and are not referred to by singular terms). This difference has important implications for the logical analysis of causal statements. To illustrate, consider the following statements:

(iii) The stabbing of Caesar by Brutus caused the death of Caesar.

(iv) The fact that Brutus stabbed Caesar caused the fact that Caesar died.

\footnotetext{
Footnote 4 continued

the focus of attention in the debates on slingshot arguments towards Gödel's variant. Causal slingshots, however, are commonly constructed along the lines of Davidson, cf. e.g. Føllesdal (1966), Anscombe (1969), Cummins and Gottlieb (1972), Mackie (1974), Williamson (1976), Levin (1976), Dale (1978), Horgan (1978, 1982), Bennett (1988), Mellor (1995), Koons (2000). The only Gödelian causal slingshots I know of can be found in Widerker (1985) and in the work of Neale. For a comprehensive overview over the literature on all variants of slingshots cf. Neale (2001).

5 Reichenbach (1947, § 48), for instance, proposes to use the notions of event and fact synonymously. Kim (1973) analyzes events as property exemplifications by objects at times, which is identical to Mellor's account of facts (Mellor 1991, pp. 203-204). Comparably, Taylor (1985, Chap. 4) takes events to be a species of facts, which he essentially spells out on a par with Mellor. Or Baylis (1948) contends that facts are particulars, which coincides with Davidson's (1967) view concerning events.
} 
In light of the above contrast between events and facts, (iii) turns out to be an atomic statement featuring the binary predicate "... causes/d ..." that relates two singular terms referring to token events, whereas (iv) constitutes a molecular statement governed by the sentential connective "The fact that ... causes/d the fact that ..." which concatenates two fact reporting closed sentences. The adequacy of this analysis, of course, is contestable, and, as this paper will show, one way to immunize a fact theory of singular causation against slingshot arguments indeed consists in contesting the adequacy of that analysis. Yet, for the time being, let us assume that (iv) is not an atomic statement composed of the binary predicate "... causes/d ..." which relates fact denoting definite descriptions as "the fact that Brutus stabbed Caesar" and "the fact that Caesar died". ${ }^{7}$ Rather, let us presume that a definite description is of the form $\imath x F x$, or colloquially "the ... such that ... is $F$ ". The 'that'-clause in "the fact that Brutus stabbed Caesar", however, states the fact and does not predicate anything of it-it does not feature a variable running over facts.

Furthermore, let us presuppose that events have a spatiotemporal locality, whereas facts are not located in space and time. The fact that Neil Armstrong stepped onto the surface of the moon on July 20, 1969, is not located on the lunar surface in 1969. In contrast, the first human step onto the surface of the moon is an event which took place on the lunar surface on July 20, 1969.

While representatives of the event camp widely agree on the categorization of events as atomic or complex particulars, ${ }^{8}$ there is considerable disagreement with respect to the ontological categorization of facts among fact theorists. Fine (1982, p. 52) broadly distinguishes between three types of conceptions of fact: one holds facts to be the truth of a proposition or statement, another identifies facts with true propositions or statements, and the third views facts to "be structured entities or complexes, built up in certain characteristic ways from their constituents". 9 Fine calls the first two conceptions propositional and the third worldly. Causal intuitions are clear in one respect: causal dependencies do not subsist between any kinds of propositional or linguistic entities. Therefore, the first of Fine's categories of fact conceptions is unsuited for a theory of causation. The debate over event versus fact causation, i.e. over whether statements of type (iii) or of type (iv) constitute the primary form of expressing token-level causal dependencies, shall thus in the present context be seen as a controversy on the question whether token-level causes are spatiotemporally located particulars called "events" or undated worldly facts.

\footnotetext{
${ }^{6}$ Some event theorists, as e.g. Schaffer (2005), take the causal relation to involve more than just two arguments. These complications, however, are of no relevance for our current purposes.

7 The majority of fact theorists-especially friends of fact causation-agree with this analysis. Some, however, do not. For instance, Oppy (1997, Sect. 5) blocks slingshot arguments by analyzing "the fact that..." in terms of a definite description which denotes a fact.

${ }^{8}$ Cf. Kanzian (2001, Chap. II.3). Notwithstanding this mutual consent with respect to the ontological categorization of events as particulars, as is well known, there is a lot of controversy in the event literature concerning identity criteria of events. This dispute can be neglected in the present context.

9 For further details on the different fact conceptions cf. (Mulligan and Correia 2007).
} 


\section{Davidson's Slingshot}

The causal slingshot argument presented by Davidson (1980, pp. 151-153) starts from the assumption that the principle of substitutivity for logical equivalents (PSLE) and the principle of substitutivity for singular terms (PSST) hold for factual causal statements. PSLE says that if $\phi$ and $\psi$ have the same truth-value relative to all systematic reinterpretations of their non-logical vocabulary, i.e. $\phi \vDash=\psi$, then, if $\Sigma(\phi)$ is a true sentence containing at least one occurrence of $\phi$, the sentence $\Sigma(\psi)$ is also true, where $\Sigma(\psi)$ results from replacing at least one occurrence of $\phi$ in $\Sigma(\phi)$ by $\psi$. PSST maintains that if two singular terms $\alpha$ and $\beta$ have the same referent, i.e. $\alpha=\beta$, then, if the sentence $\Sigma(\alpha)$ containing at least one occurrence of $\alpha$ is true, $\Sigma(\beta)$, which results from $\Sigma(\alpha)$ by replacing at least one occurrence of $\alpha$ in $\Sigma(\alpha)$ by $\beta$, is true as well. The validity of both PSLE and PSST is a necessary condition for a sentential context to be extensional, i.e. truth-functional. A sentential context is truth-functional iff material equivalents are substitutable salva veritate (s.v.), the latter being the principle of substitutivity for material equivalents (PSME).

As indicated in the introduction, the robustness of singular causation strongly supports the validity of PSLE and PSST for statements expressing causal dependencies among facts. On the basis of PSLE, of PSST, and of the standard definition of the universal class $\{x: x=x\}$, as provided by common axiomatizations of set theory featuring class abstracts-for example, the Neumann-BernaysGödel axiomatization (NBG) — , the Davidsonian causal slingshot runs as follows: ${ }^{10}$

\begin{tabular}{llll}
\hline 1 & {$[1]$} & $p \leftrightarrow q$ & A \\
2 & {$[2]$} & The fact that $p$ caused the fact that $r$. & A \\
2 & {$[3]$} & The fact that $\{x: x=x \wedge p\}=\{x: x=x\}$ caused the fact that $r$. & 2, PSLE \\
1,2 & {$[4]$} & The fact that $\{x: x=x \wedge q\}=\{x: x=x\}$ caused the fact that $r$. & 3, PSST \\
1,2 & {$[5]$} & The fact that $q$ caused the fact that $r$. & 4, PSLE \\
\hline
\end{tabular}

$\{x: x=x \wedge p\}$ designates the universal class iff $p$ is true and the null class iff $p$ is false. Hence, $\{x: x=x \wedge p\}=\{x: x=x\}$ is true whenever $p$ is true and false whenever $p$ is false. $\{x: x=x \wedge q\}$, in turn, refers to the universal class iff $q$ is true and to the null class iff $q$ is false. Since $p$ and $q$ are materially equivalent (cf. line [1]), $\{x: x=x \wedge q\}$ designates the universal class just in case $\{x: x=x \wedge p\}$ does so too and the null class whenever $\{x: x=x \wedge p\}$ refers to the null class as well. Thus, $\{x: x=x \wedge p\}$ and $\{x: x=x \wedge q\}$ are co-referring singular terms. Davidson's causal slingshot shows two things (which are not independent): first, if PSLE and PSST are accepted for factual causal contexts, not only logically, but also materially equivalent expressions are substitutable s.v. in such contexts, i.e. PSME holds as well. Accordingly, these statements are truth-functional. Second, any fact is

\footnotetext{
${ }^{10}$ There also exists a version of Davidson's slingshot that replaces class abstracts by iota-governed expressions (cf. Neale 2001, Sect. 2.6 and Chap. 8). As the discussion in the literature on event versus fact causation has focused on the version featuring class abstracts, I am going to focus on that version as well. The main findings of this section apply to either variant of Davidson's slingshot. For details on NBG cf. e.g. Fraenkel et al. (1973, Chap. II.7).
} 
just as much the cause of the fact that $r$ as any other, no matter what the fact that $r$ is. Obviously, both of these results are unacceptable.

The event camp, as already mentioned, has very much welcomed these consequences, and has used them to show that statements expressing token-level causation better not be expressed by means of factual causal statements as (iv) which are analyzed to be composed of a sentential connective concatenating fact reporting sentences. Slingshot arguments cannot be raised against event theories according to which singular causation must be expressed by statements of type (iii) that feature the predicate "... causes/d ..." which relates singular terms referring to events. In this framework, causal statements that only differ with respect to coreferring singular terms can unproblematically be claimed to express the same causal dependency. Moreover, if definite descriptions are understood referentially, any expressions - not only logically equivalent ones-may be substituted s.v. in definite descriptions as long as the latter continue to refer to the same events. The question as to the truth-functionality of eventive causal statements does not emerge in the first place. Slingshots are only aimed at theories that hold statements or sentences to state or by some means stand for extralinguistic entities.

By advancing causal slingshots, advocates of event causation intend to present their rivals of the fact causation camp with a dilemma: the latter either (A) have to subscribe to the truth-functionality of causal statements or (B) they have to reject the validity of PSLE or of PSST for causal statements which implies that their accounts cannot reproduce the robustness of singular causation. The majority of fact theorists agree that Davidson's slingshot is a valid argument, and, in consequence, acknowledge that a choice must be made between (A) and (B). As horn (A) of the dilemma would radically trivialize all accounts of fact causation, fact theorists that accept the validity of Davidson's argument all buy into horn (B). They hence reject the applicability of one or both of the inference principles to factual causal contexts. ${ }^{11}$ Moreover, a number of fact theorists additionally attempt to back up that rejection with arguments intended to show that, even though inferences as (i) and (ii) prima facie seem to be informally valid, on close inspection at least one of them is not. ${ }^{12}$ That is, they hold that, contrary to first appearances, causal dependencies indeed hinge on which among multiple logically equivalent expressions or coreferring singular terms are used to express them. These arguments, however, have not even among fact theorists lead to a consensus as to which inference principle is to be rejected nor have they convinced the event causation camp that singular causation is not robust after all. ${ }^{13}$

In light of complications of this sort, a minority of fact theorists argue that both PSLE and PSST hold for singular causal statements but that these inference principles are not properly applied in Davidson's slingshot. Cummins and Gottlieb

\footnotetext{
11 Cf. e.g. Anscombe (1969), Williamson (1976), Mellor (1991, 1995), Barwise and Perry (1996), Bennett (1988), Koons (2000).

12 Cf. e.g. Anscombe (1969), Barwise and Perry (1996), Mellor (1995).

13 While Mellor (1991, 1995) rejects PSST and endorses PSLE for causal statements, Koons (2000, pp. 35-36) rejects PSLE and endorses PSST. Proponents of event causation that uphold the robustness of singular causation - or, rather, the extensionality of singular causation which is the analogue of robustness for eventive causal statements_are e.g. Levin (1976), Kistler (1999), or Schaffer (2005).
} 
(1972), for instance, claim that, depending on whether classes are seen as objects to which reference can be made by use of class abstracts, either PSLE or PSST is fallaciously applied in Davidson's argument. On the one hand, if classes are not taken to be objects to which reference is made by class abstracts, class identity requires a contextual definition as given in (1).

$$
\{x: F x\}=\{x: G x\}=_{d f} \forall x(F x \leftrightarrow G x)
$$

Against the background of (1), $\{x: x=x \wedge p\}=\{x: x=x\}$ in line [3] of the Davidsonian slingshot is not to be read in terms of an identity statement which relates co-referring singular terms and to which PSST could be applied. Rather, $\{x: x=x \wedge p\}=\{x: x=x\}$ is a mere shorthand for $\forall x(x=x \wedge p \leftrightarrow x=x)$ which does not speak about classes and to which PSST is inapplicable. On the other hand, if classes are understood as objects to which reference can be made by means of class abstracts, the application of PSLE in Davidson's slingshot is claimed to be erroneous by Cummins and Gottlieb. They hold that on the basis of a referential interpretation of class abstracts $\{x: x=x \wedge p\}=\{x: x=x\}$ implies the existence of the universal class, whereas $p$ has no such implications. ${ }^{14}$ Thus $p$ and $\{x: x=$ $x \wedge p\}=\{x: x=x\}$ are not logically equivalent, which renders the step from line [2] to line [3] fallacious. Cummins and Gottlieb conclude that on either interpretation of class abstracts, the Davidsonian slingshot is invalid.

Proponents of slingshots, however, are very clear about the fact that they draw on a referential interpretation of class abstracts. Davidson (1980, p. 153) explicitly treats class abstracts as singular terms. Or Church (1943, pp. 302-303), who advances an analogous slingshot, unmistakably indicates that his argument presupposes a symbolism according to which class abstracts are primitive symbols, i.e. noneliminable by contextual definitions. The Davidsonian causal slingshot not only presupposes that PSLE and PSST hold for causal statements but, moreover, that class abstracts are referring symbols that are not contextually eliminated. This presupposition, which is for example satisfied in Bernays' (1958) set theory, ${ }^{15}$ guarantees that line [3] indeed features singular terms referring to classes. The subsequent application of PSST to that line is therefore perfectly sound. Furthermore, the existence of the universal class is a theorem of the NBG axiomatization of set theory (cf. e.g. Fraenkel et al. 1973, pp. 123-124). Hence, contrary to Cummins and Gottlieb, as any statement $p$ implies a theorem, $\{x: x=x \wedge p\}=\{x: x=x\}$ and $p$ are straightforwardly mutually derivable from each other and, thus, are logically equivalent. Relative to a suitable axiomatization of set theory the validity of Davidson's slingshot stands on solid ground.

Even though objections to the validity of the slingshot along the lines of Cummins and Gottlieb (1972) cannot be considered successful, they raise an important follow-up question. The slingshot is an essentially formal argument which-in case of Davidson's variant-requires a set-theoretic formalism that meets very specific syntactic constraints. While there undoubtedly exist set theories

\footnotetext{
${ }^{14}$ Cummins and Gottlieb (1972) discuss a version of Davidson's slingshot that uses the null class instead of the universal class, but that difference is of no significance for our purposes.

15 In contrast, e.g. Whitehead and Russell (1962) provide a contextual definition of class abstracts that allows for their elimination (cf. *20.3).
} 
that meet these constraints, it is, nonetheless, an open question whether such settheoretic formalisms allow for an adequate formal reproduction of statements expressing worldly facts as are involved in singular causal statements. In order for Davidson's slingshot to have ramifications for theories of fact causation, the adequacy of its formal apparatus must first be established. Unfortunately, though, proponents of the slingshot have generally taken that adequacy for granted without explicitly justifying it. That is, what is questionable is not whether class abstracts can be understood as primitive symbols or whether $p$ and $\{x: x=x \wedge p\}=\{x$ : $x=x\}$ are logically equivalent relative to a suitable system of set theory, but whether factual causal contexts are adequately formally reproducible if the notions of class abstraction and logical equivalence are borrowed from a set theory that meets the constraints of the slingshot. While symbolisms drawing on NBG have proven to be very profitably applicable, for instance, to the formalization of statements of number theory, it is far from evident that they are also suited for reproducing statements about worldly facts. The remainder of this section is therefore going to investigate whether fact theorists can possibly reject the Davidsonian slingshot by casting doubts on the adequacy its formal apparatus. We shall find that there are indeed ways to do so.

To make things concrete, let us presume that the assumption in line [2] of the Davidsonian argument stands for the causal statement (iv). Accordingly, $p$ represents:

$\left(\mathrm{S}_{1}\right)$ Brutus stabbed Caesar.

Then, an understanding of class abstracts as primitive terms yields that $\{x: x=$ $x \wedge p\}=\{x: x=x\}$ is to be read in terms of: ${ }^{16}$

$\left(\mathrm{S}_{2}\right)$ The class such that its elements are identical to themselves and Brutus stabbed Caesar is identical to the class such that its elements are identical to themselves.

Advocates of Davidson's slingshot simply presuppose that $p$ and $\{x: x=x \wedge p\}=$ $\{x: x=x\}$ are adequate formal representations of $\left(\mathrm{S}_{1}\right)$ and $\left(\mathrm{S}_{2}\right)$, respectively. But are they really?

To answer this question we have to turn to accounts of adequate formalization, as e.g. developed by Sainsbury (2001), Brun (2004), or Baumgartner and Lampert (2008). Such studies provide a number of criteria for the adequacy of formalizations, but they do not agree on all of them. To one adequacy criterion, however, all available studies subscribe: an adequate formalization must be correct. Concisely put, correctness amounts to the following: the formalization $\Phi$ of a statement $A$ is correct iff whatever formally follows from $\Phi$ informally follows from $A$, and whatever formally implies $\Phi$ informally implies $A$. Correctness is necessary but not sufficient for the adequacy of formalizations. Formalization theories complement it with further criteria - to some of which we shall turn below. Correctness renders the adequacy of formalizations dependent on two notions: formal and informal inferential dependencies among formulae and statements, respectively. While formal dependence is to be understood relative to a given calculus, two statements

\footnotetext{
${ }^{16}$ For details on how to recover colloquial statements from formalizations cf. Sainsbury (2001, pp. 64-67).
} 
are said to be informally dependent if one of them is judged to necessitate the truth or falsity of the other without recourse to any formal criterion of this necessitation. For example, while $p$ and $p \wedge q$ are formally dependent, "Cameron is a mother" and "Cameron is a woman" are informally dependent.

We have seen that the Davidsonian slingshot resorts to a formalism according to which $p$ and $\{x: x=x \wedge p\}=\{x: x=x\}$ are mutually derivable from each other. That is, in order for these formalizations to be correct, $\left(S_{1}\right)$ and $\left(S_{2}\right)$ must likewise informally follow from each other. If that is judged to be case, the formal means of Davidson's slingshot can be said to be correct and, thus, to satisfy a first necessary criterion of adequate formalization. In contrast, if $\left(\mathrm{S}_{1}\right)$ and $\left(\mathrm{S}_{2}\right)$ are not informally judged to be equivalent, $p$ and $\{x: x=x \wedge p\}=\{x: x=x\}$ directly turn out to violate correctness and, thus, to be inadequate. These considerations provide the fact theorist with two strategies to rebut the formal apparatus of Davidson's slingshot: (I) if he takes statements as $\left(\mathrm{S}_{1}\right)$ and $\left(\mathrm{S}_{2}\right)$ to be informally equivalent and, thus, $p$ and $\{x: x=x \wedge p\}=\{x: x=x\}$ to satisfy correctness, he must find a way to reject the adequacy of these formalizations based on further adequacy criteria; or (II) he shows that statements as $\left(S_{1}\right)$ and $\left(S_{2}\right)$ do not informally imply each other after all, which directly establishes that there is something fundamentally wrong with the formalizations implemented in the Davidsonian slingshot. Let us discuss the prospects of (I) and (II) in turn.

(I) If the truth of $\left(S_{2}\right)$ is taken to depend only on the truth of $\left(S_{1}\right)$ and vice versa, $\left(\mathrm{S}_{1}\right)$ and $\left(\mathrm{S}_{2}\right)$ are judged to be informally equivalent. That means the set theoretic supplement in $\left(\mathrm{S}_{2}\right)$ is vacuous and, therefore, irrelevant to the truth conditions of $\left(S_{2}\right)$. $\left(S_{2}\right)$ states neither more nor less than $\left(S_{1}\right)$. Of course, countless further statements express the same proposition as $\left(S_{1}\right)$ and $\left(S_{2}\right)$ by simply concatenating "Brutus stabbed Caesar" with some vacuous sign sequence. Thus, the question emerges as to why of all the infinitely many formulae that are formally equivalent to $p,\{x: x=x \wedge p\}=\{x: x=x\}$ should be the one that adequately captures $\left(\mathrm{S}_{2}\right)$. Why should $\left(\mathrm{S}_{2}\right)$ not be formalized by any of the following formulae which are all just as correct for $\left(S_{1}\right)$ and $\left(S_{2}\right)$-given that these statements are judged to be informally equivalent: $p \wedge \forall x x=x$ or $p \wedge(q \vee \neg q)$ or, simply, $p$ ?

Obviously, all of these alternative correct formalizations would block the step from line [3] to line [4]. That is, the fact theorist embarking on strategy (I) needs to establish that the proposition expressed by $\left(\mathrm{S}_{2}\right)$ is to be adequately formalized by $p$ or by any formally equivalent expression other than $\{x: x=x \wedge p\}=\{x: x=x\}$. In return, the proponent of the Davidsonian slingshot has to show that an adequate formal representation of the proposition expressed by $\left(S_{2}\right)$ in effect requires its vacuous set theoretic surplus to be formally captured in just the way it is captured in Davidson's argument. As correctness is only necessary but not sufficient for adequate formalization, we have to look at further adequacy criteria in order to determine which side can hope to successfully argue its case.

As indicated above, however, the formalization literature has not yet reached a consensus as to further criteria. This is particularly unfortunate for our current purposes, for, as we shall see below, the adequacy of Davidson's formalizations in the end crucially hinges on which additional criteria are chosen to complement correctness. One such additional criterion is Quine's famous maxim of shallow 
analysis: "expose no more logical structure than seems useful for the deduction or other inquiry at hand". ${ }^{17}$ Formally put, the maxim states that in addition to satisfying correctness adequate formalizations $\Phi_{1}, \Phi_{2}, \ldots, \Phi_{n}$ of statements $A_{1}$, $A_{2}, \ldots, A_{n}$ must formally reproduce the informal dependencies among $A_{1}, A_{2}, \ldots, A_{n}$ but no more. Most importantly, the maxim of shallow analysis requires adequate formalizations to be minimal to the effect that they do not feature vacuous parts that are of no relevance to the truth conditions of pertaining formulae. For instance, the validity of an argument as

(v) Socrates is wise. Wisdom is a virtue. Therefore, Socrates is virtuous.

can be formally captured by merely drawing on first-order logic as done in (2). No recourse to set theoretic formalisms is called for.

$$
\begin{aligned}
& \qquad a, \forall x(F x \rightarrow G x) \vdash G a \\
& F: \ldots \text { is wise } ; G: \ldots \text { is virtuous ; } a \text { : Socrates. }
\end{aligned}
$$

In particular, the maxim of shallow analysis identifies all correct formalizations of (v) as inadequate that involve superfluous elements as the following:

$$
\begin{gathered}
F a \wedge(p \vee \neg p), \forall x(F x \wedge x=x \rightarrow G x) \vdash G a \\
\{x: x=x \wedge(F a \vee(p \wedge \neg p))\}=\{x: x=x\}, \forall x(F x \rightarrow G x) \vdash G a
\end{gathered}
$$

Let us, hence, apply the maxim of shallow analysis to Davidson's slingshot. In order to determine whether $p$ and $\{x: x=x \wedge p\}=\{x: x=x\}$ are not only correct for $\left(\mathrm{S}_{1}\right)$ and $\left(\mathrm{S}_{2}\right)$ but moreover shallow, the purposes these formalizations are supposed to serve in the slingshot must be clarified. If $p$ and $\{x: x=x \wedge p\}=\{x$ : $x=x\}$ are interpreted in terms of $\left(\mathrm{S}_{1}\right)$ and $\left(\mathrm{S}_{2}\right)$ and $r$ is taken to stand for "Caesar died", lines [2] and [3] of Davidson's slingshot read as follows:

$\left(S_{[2]}\right)$ The fact that Brutus stabbed Caesar caused the fact that Caesar died.

$\left(\mathrm{S}_{[3]}\right)$ The fact that the class such that its elements are identical to themselves and Brutus stabbed Caesar is identical to the class such that its elements are identical to themselves caused the fact that Caesar died.

The purpose of the transition from line [2] to line [3] is to show that $\left(S_{[2]}\right)$ implies $\left(\mathrm{S}_{[3]}\right)$, or differently, to show that, if $\left(\mathrm{S}_{1}\right)$ states a cause of Caesar's death, so does $\left(\mathrm{S}_{2}\right)$. If $\left(\mathrm{S}_{1}\right)$ and $\left(\mathrm{S}_{2}\right)$ are informally judged to be equivalent, the vacuous set theoretic surplus in $\{x: x=x \wedge p\}=\{x: x=x\}$ contributes nothing whatsoever to achieve this goal. Therefore, that formalization can be directly claimed to violate the maxim of shallow analysis. Moreover, the slingshot demonstrates that if this redundant logical structure is introduced into a factual causal context, as is done on line [3], a consequence is rendered formally derivable that does not informally follow from verbalizations of lines [1] and [2]. "Brutus stabbed Caesar if and only if the Titanic sank" and "The fact that Brutus stabbed Caesar caused the fact that Caesar died" do not informally imply "The fact that the Titanic sank caused the fact

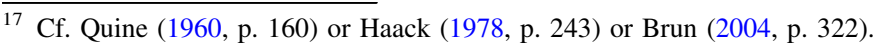


that Caesar died" - as the first two statements are true, while the third is false. Accordingly, it can be argued that to adequately formally reproduce the informal (in)dependencies among statements featuring factual causal contexts, these contexts must be formalized shallowly. Against the background of Quine's maxim, the grammatical surface of $\left(\mathrm{S}_{2}\right)$ is radically misleading as to its logical form: $\left(\mathrm{S}_{2}\right)$ is adequately formalized by $p$. The friend of fact causation can thus reject the adequacy of the formal means employed in the Davidsonian slingshot by drawing on a maxim of adequate formalization promoted by Quine who, ironically, is one of the most eager defenders of slingshot arguments.

However, the adequacy of formalizations does not necessarily have to be assessed by drawing on the maxim of shallow analysis. There are other criteria available in the literature which could be argued to be preferable. Instead of requiring adequate formalizations to be shallow, correctness can be complemented by so-called surface maxims, which, roughly, stipulate that adequate formalizations must be maximally similar to the syntactic and grammatical surface of formalized statements. ${ }^{18}$ Even though the grammatical surface of natural language is often misleading as to underlying logical forms, ${ }^{19}$ surface maxims determine that of all correct formalizations that one is adequate which adheres most closely to that surface. We have already seen that if $\left(\mathrm{S}_{1}\right)$ and $\left(\mathrm{S}_{2}\right)$ are judged to be informally equivalent, both $p$ and $\{x: x=x \wedge p\}=\{x: x=x\}$ are correct. Clearly, if $p$ is taken to represent "Brutus stabbed Caesar", $p$ is maximally similar to $\left(\mathrm{S}_{1}\right)$ and $\{x: x=x \wedge p\}=\{x: x=x\}$ is maximally similar to $\left(\mathrm{S}_{2}\right)$. That is, the proponent of the slingshot can advance surface maxims to justify the adequacy of its formal means.

The maxim of shallow analysis and surface maxims apparently identify different formalizations as adequate. Correspondingly, not both of these incompatible criteria can be chosen to complement correctness. They represent different basic approaches to logical formalization. Whoever requires adequate formalizations to be shallow sees the core function of formalizing a statement in rendering its truth conditions (or inferential context) maximally transparent. Against this background, all features of a statement that are not relevant to its truth conditions, as vacuous components, should be left out of formal reproductions because they unnecessarily disguise truth conditions. In contrast, if surface similarity is demanded of formalizations, transparency with respect to the natural language syntax of formalized statements is given preference over transparency with respect to truth conditions.

If the fact theorist rejects the slingshot on grounds of the maxim of shallow analysis and the event theorist endorses the slingshot on grounds of surface maxims, the debate over fact and event theories of causation turns into a debate as to what is the proper theory of adequate formalization. The central question now becomes: is transparency with respect to truth conditions more important than surface similarity or is it the other way around? It is far from clear what the answer to this question is going to be or whether there is even going to be a definite answer. In any case, what is of greatest importance to the fact theorist is that there is an account of adequate formalizationone professed by an important supporter of slingshots - that casts justified doubts on

${ }^{18}$ Cf. e.g. Brun (2004, Chap. 12) or Baumgartner and Lampert (2008).

${ }^{19}$ For details on the frequently cited misleading form thesis cf. e.g. Brun (2004, Chap. 7.1). 
the adequacy of the formalizations resorted to in a Davidsonian slingshot. As long as there is no decisive argument substantiating that the vacuous surplus in $\left(S_{2}\right)$ is to be formally represented by drawing on a set theoretic formalism featuring primitive class abstracts, the Davidsonian slingshot can rightly be claimed to have the air of a formal gimmick that cannot be seen to press the fact theorist into any kind of dilemma.

(II) There is another way to refute Davidson's slingshot by casting doubts on the adequacy of resorted to formalizations. Contrary to what has been said above, it could be held that statements as $\left(\mathrm{S}_{1}\right)$ and $\left(\mathrm{S}_{2}\right)$ are not informally equivalent after all. Then, every formalization of $\left(S_{1}\right)$ and $\left(S_{2}\right)$ by formally equivalent expressions can be rejected on grounds of being incorrect and, therefore, inadequate. How could the informal nonequivalence of $\left(S_{1}\right)$ and $\left(S_{2}\right)$ be substantiated? By predicating a property of an object (or, more specifically, a person) $\left(S_{1}\right)$ unquestionably states a worldly fact. In contrast, $\left(\mathrm{S}_{2}\right)$ is a statement about the alleged identity of two classes. In accordance with e.g. Wittgenstein, it could be claimed that identity can, at best, be meaningfully predicated of co-referring singular terms, in which case identity statements are to be read as rules that allow for the mutual substitution of the two terms connected by "=" (cf. Wittgenstein 1995, $\S \S 4.241-4.243,6.23$ ). Thus, with recourse to Wittgenstein the fact theorist could back up his rejection of the informal equivalence of $\left(S_{1}\right)$ and $\left(S_{2}\right)$ somehow along the following lines: $\left(S_{1}\right)$ states a worldly fact, whereas $\left(\mathrm{S}_{2}\right)$ is a rule that allows for the substitution of the expressions on both sides of the identity predicate. In consequence, it does not state a worldly fact, and hence cannot be claimed to be informally equivalent to $\left(S_{1}\right)$.

The proponent of the slingshot will of course endorse the informal equivalence of statements as $\left(S_{1}\right)$ and $\left(S_{2}\right)$, for instance, by denying that $\left(S_{2}\right)$ is a statement expressing the substitutability of two terms, for no terms are even mentioned in $\left(\mathrm{S}_{2}\right)$. Moreover, even if $\left(S_{2}\right)$ could be seen as a statement about two singular terms, the proponent of the slingshot could ask for some additional argument as to why the latter alleged feature of $\left(S_{2}\right)$ should foreclose the informal equivalence of $\left(S_{1}\right)$ and $\left(S_{2}\right)$. All that is needed for informal equivalence is that the two statements have coinciding truth conditions, and this seems to be the case for $\left(\mathrm{S}_{1}\right)$ and $\left(\mathrm{S}_{2}\right)$, regardless of the fact that $\left(\mathrm{S}_{2}\right)$ might be taken to be a statement about singular terms while $\left(\mathrm{S}_{1}\right)$ speaks about Brutus. $\left(\mathrm{S}_{1}\right)$ and $\left(\mathrm{S}_{2}\right)$ are both true iff Brutus stabbed Caesar.

Questions concerning informal equivalencies cannot conclusively be decided argumentatively. Answers to such questions essentially hinge on different readings of natural language statements which normally are ambiguous enough to allow for a host of different readings. This means that using strategy (II) to render Davidson's slingshot dubious, in the end, brings about another argumentative standoff, just as does strategy (I). Essentially, however, that is all the fact theorist aiming to avoid the slingshot dilemma needs. The adequacy of the formal means employed in Davidson's slingshot depends on what account of adequate formalization is adopted and on what informal dependencies are taken to subsist among pertaining statements. Neither of these questions is easily determinately answered. After all, the fact theorist can either give preference to the maxim of shallow analysis when it comes to assessing the adequacy of formalizations or he can deny the informal equivalence of $\left(S_{1}\right)$ and $\left(S_{2}\right)$. The proponent of the slingshot can reject both of these strategies to refute the slingshot, yet such rejections do not stand on firm theoretical ground that would be 
independent of the question as to how the conclusiveness of the slingshot is evaluated. Put differently, whoever takes the formal apparatus used in the Davidsonian slingshot to be unproblematic evaluates the adequacy of correct formalizations by drawing on surface maxims and takes $\left(\mathrm{S}_{1}\right)$ and $\left(\mathrm{S}_{2}\right)$ to be informally equivalent. Whoever sees the Davidsonian slingshot as a formal gimmick that falls short of revealing anything interesting about causal statements professes an account of adequate formalization that endorses the maxim of shallow analysis or denies the informal equivalence of $\left(\mathrm{S}_{1}\right)$ and $\left(\mathrm{S}_{2}\right)$. The whole debate between fact and event theorists then ends in a questionbegging stalemate. Contrary to the proponent of the slingshot, the fact theorist, of course, will not hesitate to welcome that standoff, for an argumentative standoff does not have a lot of power in forcing him into having to choose between (A) and (B). Pending a compelling resolution of the standoff, the fact theorist can just refuse to conclude anything from the Davidsonian slingshot.

Irrespective of how this stalemate is ultimately resolved, this section has shown that the validity of the Davidsonian slingshot essentially hinges on the exact syntactic form in which a vacuous surplus added to an informative statement is formally expressed. This finding alone suffices to give the Davidsonian slingshot the air of a formal gimmick. That the ontology of causation should, in the end, be determined by the logical form ascribed to a vacuous supplement added to a fact reporting statement seems doubtful, to say the least.

\section{Gödel's Slingshot}

Rejecting the Davidsonian slingshot either on route (I) or (II) does not yet guarantee that the advocate of fact causation can successfully avoid the slingshot dilemma. In (1995) and (2001) Neale has recalled attention to an often neglected slingshot argument that is due to Gödel (1944) and that is not as easily rejectable for being a formal gimmick. In fact, we shall see in this section that if subject-predicate statements are taken to be descriptively analyzable, the formal apparatus of Gödel's slingshot stands on solid ground. Moreover, if descriptive analyzability is dismissed (cf. e.g. Strawson 1950), one of the inference principles of Gödel's argument turns out to be invalid, which prohibits a corresponding account of fact causation from adequately capturing the robustness of singular causation. That is, while it is possible to both cast doubts on the formalizations of Davidson's argument and avoid the slingshot dilemma, challenging the formal apparatus of Gödel's slingshot pushes the fact theorist into horn (B) of that dilemma.

As is well known, a descriptive analysis of subject-predicate statements allows for rephrasing statements like $F a$ in terms of " $a$ is the $x$ such that $x=a$ and $F x$ ", or, more colloquially, in terms of " $a$ is the thing which is $F$ ". Both relative to a Russellian quantificational understanding of definite descriptions and iota-expressions, respectively, and relative to a suitable referential interpretation, expressions of type (5) and (6) are logically equivalent (cf. Neale 2001, Chaps. 9, 10). 


$$
a=\imath x(x=a \wedge F x)
$$

Gödel's slingshot then replaces PSLE by a more restricted substitution rule that no longer allows for a substitution s.v. of any logical equivalents within factual contexts, but only of logical equivalents as (5) and (6), which Neale accordingly dubs Gödelian equivalents. Gödel (1944, p. 129) says that (5) and (6) "mean the same thing". Moreover, other than, say, $\{x: x=x \wedge F a\}=\{x: x=x\}$, which is logically equivalent to (5) as well, (6) does not express a vacuous surplus that is irrelevant to its truth conditions. (6), just like (5), does not speak about anything else than the particular $a$, and it says nothing over and above $a$ being $F$. Hence, (5) and (6) - even against the background of Leibnizian identity criteria for facts-state the same fact, provided, of course, that one of them states a fact. Even if logical equivalents might not generally be substitutable in factual contexts, expressions of type (5) and (6) are assumed to be thus substitutable by Gödel. Neale labels this substitution rule -CONVERSION and abbreviates it by 1 -CONV. Furthermore, Gödel's argument presupposes the substitutability of definite descriptions and names referring to the same particular. That is, whenever for any two definite descriptions $\imath x \phi$ and $\imath x \psi: \imath x \phi=\imath x \psi$, then, $\imath x \phi$ and $\imath x \psi$ are substitutable s.v. in factual contexts. Likewise, whenever for any definite description $\imath x \phi$ and any name $\alpha: \imath x \phi=\alpha$, then, $\imath x \phi$ and $\alpha$ are substitutable s.v. Neale refers to this rule as i-Substitution, or 1 -SUBs for short.

Given 1 -CONV and 1 -SUBS, the Gödelian type slingshot-as reconstructed by Neale-runs as follows: ${ }^{20}$

\begin{tabular}{llll}
\hline 1 & {$[1]$} & $F a$ & $\mathrm{~A}$ \\
2 & {$[2]$} & $a \neq b$ & $\mathrm{~A}$ \\
3 & {$[3]$} & $G b$ & $\mathrm{~A}$ \\
1 & {$[4]$} & $a=\imath x(x=a \wedge F x)$ & 1,1 -CONV \\
2 & {$[5]$} & $a=\imath x(x=a \wedge x \neq b)$ & 2,1 -CONV \\
2 & {$[6]$} & $b=\imath x(x=b \wedge x \neq a)$ & 2,1 -CONV \\
3 & {$[7]$} & $b=\imath x(x=b \wedge G x)$ & 3,1 -CONV \\
1,2 & {$[8]$} & $\imath x(x=a \wedge F x)=\imath x(x=a \wedge x \neq b)$ & $4,5,1$-SUBS \\
2,3 & {$[9]$} & $2 x(x=b \wedge G x) \imath x(x=b \wedge x \neq a)$ & $6,7,1$-SUBS \\
10 & {$[10]$} & The fact that $F a$ caused the fact that $p$. & $\mathrm{A}$ \\
10 & {$[11]$} & The fact that $a=\imath x(x=a \wedge F x)$ caused the fact that $p$. & 10,1 -CONV \\
$1,2,10$ & {$[12]$} & The fact that $a=\imath x(x=a \wedge x \neq b)$ caused the fact that $p$. & $11,8,1$-SUBS \\
$1,2,10$ & {$[13]$} & The fact that $a \neq b$ caused the fact that $p$. & 12,1 -CONV \\
$1,2,10$ & {$[14]$} & The fact that $b=\imath x(x=b \wedge x \neq a)$ caused the fact that $p$. & 13,1 -CONV \\
$1,2,3,10$ & {$[15]$} & The fact that $b=\imath x(x=b \wedge G x)$ caused the fact that $p$. & $14,9,1$-SUBS \\
$1,2,3,10$ & {$[16]$} & The fact that $G b$ caused the fact that $p$. & 15,1 -CONV \\
\hline
\end{tabular}

\footnotetext{
${ }^{20}$ Cf. Neale (2001, pp. 183-184). In its original form the Gödelian slingshot is not tailored to the causal connective. For the present inquiry, though, nothing more is of interest.
} 
The consequences of the Gödelian slingshot essentially coincide with what follows from Davidson's argument. Provided that 2 -CONV and 2 -SUBS are valid for factual contexts as the one of line [10], it follows that PSME is valid for such contexts, and thus that the latter are truth-functional, moreover, that any fact caused any other fact.

Prima facie, it might be thought that the same resorts are open to the advocate of fact causation as in case of Davidson's slingshot: either he accepts the validity of Gödel's argument and, in consequence, chooses horn (B) of the slingshot dilemma by rejecting the applicability of one of $\iota$-CONV and $\lambda$-SUBS to factual causal statements, or he discards the formal apparatus of the argument for reasons of inadequacy and thereby avoids the slingshot dilemma. Yet, whereas in case of Davidson's slingshot the questions as to the adequacy of the formal apparatus, on the one hand, and to the applicability of PSLE and PSST to factual causal contexts, on the other, can be separated, the two questions are closely intertwined in case of Gödel's argument. The latter crucially presupposes a descriptive analyzability of subject-predicate statements. Accepting this presupposition amounts to both endorsing the adequacy of the formalizations implemented in the Gödelian slingshot and to endorsing the applicability of 2 -CONV to subject-predicate statements.

Whoever subscribes to a descriptive analyzability of subject-predicate statements claims that the following statements are informally equivalent:

$\left(\mathrm{S}_{5}\right) \quad$ Brutus stabbed Caesar.

$\left(\mathrm{S}_{6}\right) \quad$ Brutus is the one who stabbed Caesar.

Of course, these informal equivalencies can be rejected. ${ }^{21}$ However, Russell's descriptive analysis of subject-predicate statements, for instance, has proven very valuable as regards empty predications or true negative existential statements. Moreover, by denying that statements of type $\left(S_{5}\right)$ and $\left(S_{6}\right)$ are informally equivalent one also denies that the two statements express identical facts. Claiming that $\left(S_{5}\right)$ and $\left(S_{6}\right)$ do not state the same fact has consequences for factual causal statements that are far more problematic than claiming that $\left(\mathrm{S}_{1}\right)$ and $\left(\mathrm{S}_{2}\right)$ are not informally equivalent. Since a statement expressing class identity as $\left(\mathrm{S}_{2}\right)$ does not appear to state a cause at all, it indeed is questionable that $\left(S_{1}\right)$ and $\left(S_{2}\right)$ state the same cause of Caesar's death. In contrast, if one of $\left(S_{5}\right)$ and $\left(S_{6}\right)$ states a cause of Caesar's death, the other expresses the same cause of that effect. That means by denying the informal equivalence of $\left(\mathrm{S}_{5}\right)$ and $\left(\mathrm{S}_{6}\right)$ the fact theorist's account of singular causation can no longer reproduce the robustness of singular causation.

If, in view of this problem, the fact theorist does not contest the informal equivalence of $\left(S_{5}\right)$ and $\left(S_{6}\right)$, it follows that the equivalent formalizations (5) and (6) are correct. Furthermore, neither (5) nor (6) features a vacuous surplus that would have to be eliminated in order to meet the maxim of shallow analysis. On the contrary, to somebody professing a descriptive analysis of subject-predicate statements an expression as (6) renders the logical form of such statements more transparent than a formula as (5). (6) might thus even be claimed to be preferable

\footnotetext{
${ }^{21}$ Cf. Strawson (1950). For a detailed discussion of the Russell-Strawson debate concerning the proper logical analysis of subject-predicate statements cf. Baumgartner (forthcoming).
} 
over (5). Moreover, it is plain that (5) and (6) each are maximally similar to the syntactical surface of $\left(S_{5}\right)$ and $\left(S_{6}\right)$, respectively. Thus, given that, in light of the robustness of singular causation, one accepts the descriptive analyzability of subject-predicate statements in causal contexts, the formal apparatus of Gödel's argument is perfectly adequate. In addition, endorsing the overall descriptive analyzability of subject-predicate statements in factual contexts amounts to endorsing the validity of 1 -CONV for such contexts. 1 -CONV is nothing but a formal expression of the descriptive analyzability of subject-predicate statements. All in all thus, the adequacy of the formalizations resorted to in Gödel's slingshot and the validity of 1 -CONV stand and fall together. While the fact theorist can rebut Davidson's slingshot and, at the same time, avoid the slingshot dilemma by casting doubts on the formal apparatus resorted to in that argument, there is no such innocuous way around Gödel's slingshot. Challenging the formal apparatus of Gödel's slingshot amounts to challenging one of its inference principles which, in turn, amounts to conceding that causing and caused facts can be multiplied by descriptively rephrasing corresponding fact reporting statements.

The representative of fact causation not willing to dismiss the descriptive rephrasability of subject-predicate statements and, hence, the validity of 1 -CONV can only avoid having to concede that, on his account, any fact causes any other fact, if he denies the validity of 1 -SuBs for factual contexts. And indeed, such a rejection of 1-SUBS receives weighty support from Russell. According to Russell's theory of descriptions, definite descriptions are 'incomplete symbols' that do not refer to anything. Hence, questions as to the co-reference of definite descriptions do not arise in the first place. ${ }^{22}$ Definite descriptions never occur in isolation, but only in broader sentential contexts, where, according to Russell, they get a quantificational and not a referential interpretation. In order to illustrate Russell's point consider the following expressions:

$$
\begin{gathered}
F a \\
F \mathfrak{x} x(x=a) \\
\exists x(x=a \wedge \forall y(y=a \rightarrow y=x) \wedge F x) \\
\exists x(A x \wedge \forall y(\mathrm{~A} y \rightarrow y=x) \wedge F x)
\end{gathered}
$$

While (7) is a subject-predicate statement that predicates of the particular $a$ that it is $F$, (8) does not speak of a specific particular, but is an existentially quantified statement to be understood in terms of (9). In (9) the name $a$ only occurs in the context "= $a$ " from which, as Russell suggests in Russell (1937/1992, p. 152), it can be straightforwardly eliminated by expressing " $=a "$ by means of an ordinary

\footnotetext{
22 Cf. Russell (1977, pp. 244-246, 253-254) also Neale (2001, p. 167). In view of Gödel's slingshot, the fact that 2 -SUBS is invalidated by Russell's theory of descriptions is sometimes interpreted as additional evidence for the adequacy of the theory.
} 
unary predicate $A$ that only applies to one object, viz. $a$. Thus, (9) can be spelled out in terms of (10).

Carried over to the factual context at hand, despite their indubitable close connection, (7) and (8), following Russell, do not state the same fact, for in Russell's terminology (7) expresses a particular fact, whereas (8) states a general fact (cf. Russell 1977, pp. 183-184, 234-235). That means if the friend of fact causation, on a par with Russell, treats definite descriptions as non-referring incomplete symbols that only appear in statements that are to be read as quantified expressions like (10), he can reject the validity of $\iota$-SUBS for factual contexts without thereby rejecting the substitutability s.v. of co-referring singular terms (PSST). To him 1 -SUBS is invalid because definite descriptions do not refer in the first place, and, therefore, there cannot be any co-referring definite descriptions.

However, in what follows it shall be shown that even though a quantificational understanding of definite descriptions along with the rejection of 1 -SUBS allows the representative of fact causation to rebut the Gödelian slingshot and, at the same time, stick to PSST, the consequences of rejecting 1 -SUBS for contexts governed by "The fact that ... caused the fact that ..."- - to a large extent-are the same as the consequences of a refutation of PSST: an overly fine-grained analysis of causal processes that does not mirror the robustness of singular causation. Consider the following argument:

(vi) (P1) The fact that the son of Servilia Caepionis stabbed Caesar caused the fact that Caesar died.

(P2) The son of Servilia Caepionis is identical to the husband of Porcia Catonis.

(C) The fact that the husband of Porcia Catonis stabbed Caesar caused the fact that Caesar died.

In light of the robustness of singular causation, (vi) is an informally valid argument. Relative to all reinterpretations of the non-logical vocabulary embedded in the factual contexts of (vi) it holds that if the premises are judged to be true, so is the conclusion. If (P2) is true, (P1) and (C) state the same causal dependency as they pick out the same cause of the fact that Caesar died. A theory of fact causation that, based on a Russellian analysis of definite descriptions, rejects 1 -SUBS for contexts governed by "The fact that ... caused the fact that ..." and that, nonetheless, purports to account for the robustness of singular causation, has to be able to reproduce the validity of (vi) without $\iota$-SUBS. To see whether this can be done, two Russellian readings of (vi) need to be distinguished, for, as is well known, a quantificational account of definite descriptions may involve ambiguities of scope. The definite descriptions contained in (vi) can be understood to have either narrow or wide scope. ${ }^{23}$ By letting $F$ stand for “... is a son of Servilia Caepionis", $G$ for "... stabbed Caesar", $p$ for "Caesar died" and by representing the factual causal

\footnotetext{
${ }^{23}$ For more details on scope ambiguities involving definite descriptions cf. Neale (1990, Chap. 4). The possibility of interpreting definite descriptions in factual causal statements to have wide scope is normally not considered in the literature. I owe this interpretation of causal statements to Michael Gabbay.
} 
connective by “ $\mapsto$ ”, the narrow scope reading of $(\mathrm{P} 1)$ is expressible in terms of $\left(P_{1}^{n}\right)$ and the wide scope reading in terms of $\left(P_{1}^{w}\right)$.

$$
\begin{aligned}
& \exists x(F x \wedge \forall y(F y \rightarrow x=y) \wedge G x) \mapsto p \\
& \exists x(F x \wedge \forall y(F y \rightarrow x=y) \wedge(G x \mapsto p))
\end{aligned}
$$

In contrast, there are no scope ambiguities involved in (P2). By introducing $H$ for “... is a husband of Porcia Catonis", a Russellian analysis of (P2) yields:

$$
\exists x \exists y\left(F x \wedge \forall z_{1}\left(F z_{1} \rightarrow x=z_{1}\right) \wedge H y \wedge \forall z_{2}\left(H z_{2} \rightarrow y=z_{2}\right) \wedge x=y\right)
$$

Analogously to the first premise, the conclusion of (vi) allows for a narrow and a wide scope reading which are formally reproduced in $\left(C^{n}\right)$ and $\left(C^{w}\right)$, respectively.

$$
\begin{aligned}
& \exists x(H x \wedge \forall y(H y \rightarrow x=y) \wedge G x) \mapsto p \\
& \exists x(H x \wedge \forall y(H y \rightarrow x=y) \wedge(G x \mapsto p))
\end{aligned}
$$

The main formal difference between the narrow and the wide scope readings is that according to the narrow scope reading (P1) and (C) are molecular statements governed by the factual causal connective, whereas according to the wide scope reading $(\mathrm{P} 1)$ and $(\mathrm{C})$ are existentially quantified conjunctions, i.e. they turn out to be non-molecular. In consequence, the truth conditions of $\left(P_{1}^{n}\right)$ and $\left(C^{n}\right)$ fully depend on the semantics of the non-extensional connective "The fact that ... caused the fact that $\ldots$.. In $\left(P_{1}^{w}\right)$ and $\left(C^{w}\right)$, however, the factual causal connective only governs the third conjunct within the scopes of the existential quantifiers. This difference has important ramifications for the validity of the resulting formalizations of the whole argument (vi). $\left(P_{2}\right)$ states that the extensions of $F$ and $H$ comprise exactly one and the same element. As coextensional predicates can be substituted s.v. in extensional contexts, the validity of the two Russellian readings of (vi) hinges on whether $F$ and $H$ occur in extensional contexts. While that is not the case for $\left(P_{1}^{n}\right)$ and $\left(C^{n}\right), F$ and $H$ are located outside of the context governed by the intensional causal connective in $\left(P_{1}^{w}\right)$ and $\left(C^{w}\right)$. That is, $\left(P_{2}\right)$ licenses to replace $H$ for $F$ in $\left(P_{1}^{w}\right)$ which directly yields $\left(C^{w}\right)$. In sum, whereas a narrow scope analysis of (vi) generates an invalid formalization, the validity of (vi), on the face of it, seems to be formally reproducible without the use of 1 -SUBS by drawing on a wide scope reading of the definite descriptions contained in (P1) and (C):

$$
\begin{aligned}
& \left(P_{1}^{n}\right),\left(P_{2}\right) \nvdash\left(C^{n}\right) \\
& \left(P_{1}^{w}\right),\left(P_{2}\right) \vdash\left(C^{w}\right)
\end{aligned}
$$

Prima facie, it thus appears that a Russellian wide scope understanding of definite descriptions enables the friend of fact causation to block Gödel's slingshot by rejecting 1 -SUBS for factual contexts and, nonetheless, account for the validity of arguments as (vi), and hence for the robustness of singular causation. Securing fact causation against the threat posed by Gödel's slingshot without being forced into horn (B) of the slingshot dilemma, however, presupposes that $\left(P_{1}^{w}\right)$ and $\left(C^{w}\right)$ are adequate formal representations of $(\mathrm{P} 1)$ and $(\mathrm{C})$, respectively. Is that indeed the case? To answer that question we need to establish that $\left(P_{1}^{w}\right)$ and $\left(C^{w}\right)$ determine the 
same facts to be causally related as their informal counterparts (P1) and (C). Clearly, both $\left(P_{1}^{w}\right)$ and $\left(C^{w}\right)$ identify the fact that Caesar died, i.e. the fact expressed by $p$, as caused fact, which fact is also identified as caused fact by (P1) and (C). Furthermore, $\left(P_{1}^{w}\right)$ and $\left(C^{w}\right)$ analyze the definite descriptions contained in the causing facts mentioned in (P1) and (C) in terms of existentially quantified expressions whose scopes comprise both the caused fact stated by $p$ and the causal dependency $G x \mapsto p$. Spelling this wide scope out in informal terms yields that $\left(P_{1}^{w}\right)$, for example, states a causal dependency among the following facts:

Causing fact $(A)$ : the fact that there exists exactly one son of Servilia Caepionis whose stabbing of Caesar caused the fact that Caesar died.

Causing fact $(B)$ : the fact that Caesar died.

That is, $\left(P_{1}^{w}\right)$ identifies a causing fact $A$ which can only be the case, if the caused fact $B$ is the case as well-and analogously for $\left(C^{w}\right)$. The facts that can be said to be causally related by $\left(P_{1}^{w}\right)$ and $\left(C^{w}\right)$, hence, are logically dependent. In contrast, the facts claimed to be causally related by, say, $(\mathrm{P} 1)$ are the fact that the son of Servilia Caepionis stabbed Caesar and the fact that Caesar died, which are logically independent. Generally, causes and effects on token level are logically independent. Causal dependencies arise from material, not from logical dependencies. The latter cannot be interpreted causally. In accordance, semantics for factual statements expressing causal dependencies on token level, as are e.g. developed by Bennett (1988) or Mellor (1995), are of the form: "The fact that $\phi$ caused the fact that $\psi$ " is true iff $\Omega(\phi, \psi)$, where $\phi$ and $\psi$ stand for closed sentences and $\Omega$ denotes the set of conditions imposed by a pertaining theory in order for $\phi$ and $\psi$ to be causally connected. Common candidates for $\Omega$ are, for instance, that $\psi$ must be derivable from $\phi$ in combination with a causal law $\mathcal{L}$ and a causal background $S$ or that the probability/propensity/chance that $\psi$ holds is higher in the closest $\phi$-worlds than in the closest non- $\phi$-worlds. While the details of these different proposals for $\Omega$ are of no relevance for our purposes, it is of crucial importance to note that factual causal statements relate logically independent closed sentences stating the causing and the caused fact.

This constraint is straightforwardly met by the narrow scope readings of (vi). $\left(P_{1}^{n}\right)$, for example, identifies the fact that there exists exactly one son of Servilia Caepionis who stabbed Caesar as cause of the fact that Caesar died-and analogously for $\left(C^{n}\right)$. The constraint, however, is violated in the wide scope readings. In $\left(P_{1}^{w}\right)$ and $\left(C^{w}\right)$ the first expression governed by $\mapsto$ is an open sentence. Hence, the truth conditions of the third conjuncts within the scopes of the existential quantifiers of $\left(P_{1}^{w}\right)$ and $\left(C^{w}\right)$ are not determinable by means of available semantics for the factual causal connective. Moreover, as $A$ is sufficient for $B$ on mere conceptual grounds, $B$ is derivable from $A$ even without causal laws and $A$ trivially raises the probability of $B$ to 1 . Any reading of causal statements that renders causes and effects logically dependent does not adequately capture the truth conditions or logical form of such statements. Thus, definite descriptions in factual causal contexts on token level must either be interpreted referentially or, if a quantificational analysis is preferred, they must be interpreted to have narrow scope. The referential reading gives rise to the slingshot, the narrow scope reading does not 
allow for reproducing the validity of arguments as (vi). All in all, this shows that a theory of fact causation which rejects 1 -SUBS in light of Gödel's slingshot cannot account for the robustness of singular causation and, accordingly, has to buy into horn (B) of the slingshot dilemma after all.

\section{Conclusion}

Slingshot arguments are designed to press fact theorists into advancing a very finegrained notion of fact identity, according to which replacing fact reporting expressions by logical equivalents or substituting co-referring singular terms within fact reporting statements does not guarantee the identity of expressed facts. Yet, if proponents of fact causation succumb to that pressure and draw on a sufficiently fine-grained account of fact identity, their theories become incapable of doing justice to the robustness of singular causation.

This paper has shown that the advocate of fact causation can avoid this impasse in case of Davidson's slingshot by casting reasonable doubts on the formal apparatus used in that argument. Such an innocuous way around the slingshot, however, is not on hand in case of Gödel's often neglected argument. Rejecting the formal apparatus of Gödel's argument entails the invalidity of one of the inference rules used in that argument which, in turn, yields a fact theory of causation that is too fine-grained for an adequate account of the robustness of causation.

A theory of singular causation which neither implies that any token cause causes any token effect nor that singular causation is not robust cannot take causal statements to be molecular expressions governed by a sentential connective as "The fact that ... causes/d the fact that ...". Slingshots cannot be advanced against accounts that analyze statements expressing singular causal dependencies in terms of expressions featuring the predicate "... causes/d ...". Does that upshot of our discussion count against a fact ontology of singular causation? Not necessarily. A statement like "The fact that Brutus stabbed Caesar caused the fact that Caesar died" might well be analyzed as involving the predicate "... causes/d ..." which takes fact denoting singular terms like "the fact that Brutus stabbed Caesar" as arguments. As mentioned in section 2, some fact theorists-e.g. Baylis (1948) have indeed professed fact ontologies according to which facts are particulars, and others-e.g. Oppy (1997)—analyze expressions of type "the fact that ..." as definite descriptions referring to facts. Of course, fleshing out such rare sketches into a full-blown theory of fact particulars that not only avoids the slingshot but, moreover, meets the requirements of an analysis of singular causation, would require answering important questions which, due to the unorthodox character of such a proposal, have not been properly addressed in the literature. Most of all, identity criteria for fact particulars would be required that are compatible with the robustness of singular causation. Presumably, answering such questions is not straightforward. Nonetheless, there do not seem to be principled obstacles to fleshing out such a theory. After all, there exist various worked out theories of event particulars that have been effectively employed in accounts of causation. Prima facie, thus, construing fact particulars along the lines of events seems a promising 
way to go for the friend of fact causation. Clearly though, such a 'particularist' maneuver would reduce the dispute between fact and event theorists of singular causation to a mere terminological controversy. For within such a framework both sides would agree on the logical form of causal statements and on the ontological categorization of causes and effects as particulars. They would merely be at odds with respect to the label that should be assigned to the pertaining sort of particulars.

In sum, causal slingshots do not prove a fact ontology of causation to be impossible. Nonetheless, the Gödelian slingshot provides strong reasons to analyze statements expressing singular causal dependencies in terms of statements featuring the predicate "... causes/d ...". Such a logical analysis has traditionally been professed by virtually all event theorists and by only very few advocates of fact causation.

Acknowledgements My particular thanks go to Timm Lampert for countless discussions about facts and slingshots, as well as for our common work on logical formalization. Moreover, I am grateful to Michael Gabbay and to the anonymous referees of this journal for very helpful comments on earlier drafts. Finally, I thank the Swiss National Science Foundation for generous support of this work (grant PP001-114812/1).

\section{References}

Anscombe, G. E. M. (1969). Causality and extensionality. Journal of Philosophy, 66, 152-159.

Barwise, J., \& Perry, J. (1996). Semantic innocence and uncompromising situations. In A. P. Martinich (Ed.), The philosophy of language (pp. 369-381). Oxford: Oxford University Press. [originally published 1975 in midwest studies in philosophy]

Baumgartner, M. (forthcoming). Informal reasoning and logical formalization. In S. J. Conrad \& S. Imhof (Eds.), Ding und Begriff. Frankfurt a.M.: Ontos Verlag.

Baumgartner, M., \& Lampert, T. (2008). Adequate formalization. Synthese, 164, 93-115.

Baylis, C. A. (1948). Facts, propositions, exemplification and truth. Mind, 57, 459-479.

Bennett, J. (1988). Events and their names. Indianapolis: Hackett.

Bernays, P. (1958). Axiomatic set theory. Amsterdam: North-Holland.

Brun, G. (2004). Die richtige Formel. Philosophische Probleme der logischen Formalisierung. Frankfurt a.M.: Ontos Verlag.

Church, A. (1943). Review of Carnap's introduction to semantics. Philosophical Review, 52, 298-304.

Cummins, R., \& Gottlieb, D. (1972). On an argument on truth-functionality. American Philosophical Quarterly, 9, 265-269.

Dale, A. J. (1978). Reference, truth-functionality, and causal connectives. Analysis, 38, 99-106.

Davidson, D. (1967). The logical form of action sentences. In N. Rescher (Ed.), The logic of decision and action (pp. 81-95). Pittsburgh: University of Pittsburgh Press.

Davidson, D. (1980). Essays on actions and events. Oxford: Clarendon Press.

Dowe, P. (2000). Physical causation. Cambridge: Cambridge University Press.

Fine, K. (1982). First-order modal theories III-facts. Synthese, 53, 43-122.

Føllesdal, D. (1966). Quantification into causal contexts. In R. S. Cohen \& M. W. Wartofsky (Eds.), Boston studies in the philosophy of science (vol. II, pp. 263-274). New York: Humanities Press.

Fraenkel, A. A., Bar-Hillel, Y., \& Levy, A. (1973). Foundations of set theory (2nd ed.). Amsterdam: North-Holland.

Gödel, K. (1944). Russell's mathematical logic. In P. A. Schilpp (Ed.), The philosophy of Betrand Russell (pp. 123-153). New York: Tudor.

Haack, R. J. (1978). Quine's theory of logic. Erkenntnis, 13, 231-259.

Horgan, T. (1978). The case against events. The Philosophical Review, 87, 28-47.

Horgan, T. (1982). Substitutivity and the causal connective. Philosophical Studies, 42, 47-52.

Kanzian, C. (2001). Ereignisse und andere Partikularien. Paderborn: Ferdinand Schöningh. 
Kim, J. (1973). Causation, nomic subsumption, and the concept of event. Journal of Philosophy, 70, 217-236.

Kistler, M. (1999). Causes as events and facts. Dialectica, 53, 25-46.

Koons, R. C. (2000). Realism regained. An exact theory of causation, teleology, and the mind. Oxford: Oxford University Press.

Levin, M. E. (1976). The extensionality of causation and causal-explanatory contexts. Philosophy of Science, 43, 266-277.

Mackie, J. L. (1974). The cement of the universe. A study of causation. Oxford: Clarendon Press.

Massey, G. (1981). Logic and linguistics. In E. Agazzi (Ed.), Modern logic-a survey. Historical, philosophical, and mathematical aspects of modern logic and its applications (pp. 311-329). Dordrecht: Reidel.

Mellor, D. H. (1991). The singularity affecting facts of causation. In Matters of metaphysics (pp. 201224). Cambridge: Cambridge University Press.

Mellor, D. H. (1995). The facts of causation. London: Routledge.

Mulligan, K., \& Correia, F. (2007). Facts. In E. N. Zalta (Ed.), The stanford encyclopedia of philosophy. http://plato.stanford.edu/archives/win2007/entries/facts/.

Neale, S. (1990). Descriptions. Cambridge: MIT Press.

Neale, S. (1995). The philosophical significance of Gödel's slingshot. Mind, 104, 761-825.

Neale, S. (2001). Facing facts. Oxford: Clarendon Press.

Oppy, G. (1997). The philosophical insignificance of Gödel's slingshot. Mind, 106, 121-141.

Quine, W. v. O. (1960). Word and object. Cambridge: MIT Press.

Ramsey, F. P. (1927/1994). Facts and propositions. In D. H. Mellor (Ed.), Philosophical papers (pp. 3451). Cambridge: Cambridge University Press.

Reichenbach, H. (1947). Elements of symbolic logic. New York: Collier-Macmillan.

Russell, B. (1937/1992). The principles of mathematics. London: Routledge.

Russell, B. (1977). The philosophy of logical atomism. In R. Marsh (Ed.), Logic and knowledge (pp. 175281). New York: Allen \& Unwin.

Sainsbury, R. M. (2001). Logical forms (2nd ed.). Oxford: Blackwell.

Schaffer, J. (2005). Contrastive causation. Philosophical Review, 114, 327-358.

Strawson, P. F. (1950). On referring. Mind, 59, 320-344.

Taylor, B. (1985). Modes of occurrence: verbs, adverbs, and events. Oxford: Blackwell.

Whitehead, A. N., \& Russell, B. (1962). Principia Mathematica. Cambridge: Cambridge University Press.

Widerker, D. (1985). Davidson on singular causal sentences. Erkenntnis, 23, 223-242.

Williamson, J. (1976). Facts and truth. The Philosophical Quarterly, 26, 203-216.

Wittgenstein, L. (1995). Tractatus logico-philosophicus. Frankfurt a.M.: Suhrkamp. 